\title{
Erratum
}

\section{The Effect of Storage Conditions on the Permeability of Porcine Buccal Mucosa}

\author{
Jaehwi Lee', Sang Kil Lww', and Young Wook Choi ${ }^{2}$ \\ ${ }^{1}$ Department of Industrial and Physical Pharmacy, School of Pharmacy and Pharmacal Sciences, Purdue University, \\ West Lafayette, IN 47907-1336, U.S.A. and ${ }^{2}$ College of Pharmacy, Chung-Ang University, Seoul 156-756, Korea
}

The correct name is

"Jaehwi Lee', Sang Kil Lee', and Young Wook Choi"?". 